\title{
Corrigendum: Use of the Gibbs thermodynamic potential to express the equation of state in atmospheric models
}

\author{
J. Thuburn* \\ University of Exeter, Exeter, UK
}

${ }^{*}$ Correspondence to: Department of Mathematics, College of Engineering, Mathematics and Physical Sciences, University of Exeter, Exeter, EX4 4QF, UK.

Received...

The paper by Thuburn (2017) shows that in a numerical model of atmospheric fluid dynamics the equation of state can be expressed in terms of the Gibbs thermodynamic potential or Gibbs function; different equations of state can then be conveniently applied simply by changing the Gibbs function. However, the claim that the pseudo-incompressible system of Durran (1989) can be obtained in this way is incorrect.

Durran (1989) (see also Durran 2008) shows that the pseudo-incompressible system can be obtained from the compressible Euler equations by modifying only the equation of state, leaving the momentum equation unchanged. However, this is true only when the pressure gradient term in the momentum equation is wrtten as $C_{p} \theta \nabla \Pi$, where $C_{p}$ is the specific heat capacity at constant pressure, $\theta$ is potential temperature, and $\Pi$ is the Exner pressure. It is no longer true when the pressure gradient term is written as $(1 / \rho) \nabla p$, where $p$ is pressure. The pseudo-incompressible Gibbs function given by Thuburn (2017) does indeed give Durran's pseudoincompressible equation of state. However, because the pressure gradient is written as $(1 / \rho) \nabla p$, Thuburn's pseudo-incompressible system is not equivalent to Durran's. For similar reasons, the blended compressible-pseudo-incompressible equation of state implied by Thuburn's equation (68) does indeed agree with the blended equation of state of Klein and Benacchio (2016), but the corresponding fluid dynamical systems are not equivalent.

The pseudo-incompressible system used by Thuburn (2017) is not, in fact energy conserving (which rather undermines a major motivation of the Gibbs function approach). Vasil et al. (2013) discuss the modifications needed to the momentum equation in order to obtain an energy conserving pseudo-incompressible system when the pressure gradient is in $(1 / \rho) \nabla p$ form. See also Klein and Pauluis (2012).

The energy-inconsistency errors in the pseudo-incompressible buoyant bubble test case shown by Thuburn (2017) are actually rather small and are comparable to the conservation errors resulting from numerical approximations at the resolution used (approximately $100 \mathrm{~m}$ ). However, unlike the numerical errors, the inconsistency errors do not reduce as resolution is refined.

\section{References}

Durran DR. 1989. Improving the anelastic approximation. J. Atmos. Sci. 46: 1453-1461.

Durran DR. 2008. A physically motivated approach for filtering acoustic waves from the equations governing compressible stratified flow. J. Fluid Mech. 601: 365-379.

Klein R, Benacchio T. 2016. A doubly blended model for multiscale atmospheric dynamics. J. Atmos. Sci. 73: 1179-1186.

Klein R, Pauluis O. 2012. Thermodynamic consistency of a pseudoincompressible approximation for general equations of state. J. Atmos. Sci. 69: 961-968.

Thuburn J. 2017. Use of the Gibbs thermodynamic potential to express the equation of state in atmospheric models. Quart. J. Roy. Meteor. Soc. 143: 1185-1196.

Vasil GM, Lecoanet D, Brown BP, Wood TS, Zweibel EG. 2013. Energy conservation and gravity waves in sound-proof treatments of stellar interiors. II. Lagrangian constrained analysis. Astrophys. J. 773(169), doi:10.1088/0004-637X/773/2/169. 